\title{
UNIQUE CONTINUATION THEOREMS FOR SOME PARABOLIC OPERATORS
}

\author{
J. D. McMICHAEL AND D. M. OBERLIN \\ (Communicated by Barbara L. Keyfitz)
}

\begin{abstract}
We prove a unique continuation theorem for a class of differential operators containing the heat operator.
\end{abstract}

Suppose $j_{1}, \ldots, j_{n}$ and $j$ are positive integers and let $E$ be the differential operator on $\mathbf{R}^{n}$ whose symbol is

$$
\sigma(\xi)=-\left(\sum_{l=1}^{n} \xi_{l}^{2 j_{l}}\right)^{j}
$$

The purpose of this paper is to prove a unique continuation theorem for the operator $L=\partial / \partial t-E$ on $\mathbf{R}^{n+1}$.

Theorem. Let $r=\sum_{l=1}^{n}\left(2 j j_{l}\right)^{-1}+1$. Suppose $u \in C_{0}^{\infty}\left(\mathbf{R}^{n+1}\right)$ satisfies the differential inequality

$$
|L u(x, t)| \leq|V(x, t) u(x, t)|
$$

for some $V \in L^{r}\left(\mathbf{R}^{n+1}\right)$. Then $u \equiv 0$.

If $L$ is the heat operator $\partial / \partial t-\Delta$, then $r=n / 2+1$. In this case our theorem yields a conclusion analogous to [3, Corollary 1], although that result for the Schrödinger operator $i \partial / \partial t+\Delta$ requires only that $u$ vanish in a half-space. Our method of proof is also similar to that of [3] (and [2]) in that we deduce our unique continuation theorem from a Carleman estimate which is a consequence of a uniform Sobolev inequality. But while the Sobolev inequalities of [2] and [3] are based on restriction theorems for the Fourier transform, ours depends only on Young's inequality and an elementary estimate. This may reflect the fact that our operators $L$ are "less singular" than those treated in [2] and [3]. Our main task, then, will be to establish the following result.

Received by the editors February 3, 1989.

1980 Mathematics Subject Classification (1985 Revision). Primary 35L15, 42B25.

Key words and phrases. Sobolev inequalities, unique continuation. 
Lemma 1. With $L$ and $r$ as above, fix $p$ and $q$ with $1<p<q<\infty$ and $1 / p-1 / q=1 / r$. There is a constant $C=C(L, p)$ such that for any $\lambda<0$ and any $u \in C_{0}^{\infty}\left(\mathbf{R}^{n+1}\right)$ we have

$$
\|u\|_{q} \leq C\|(L-\lambda) u\|_{p} .
$$

Combining the observation

$$
e^{\lambda t} L u=(L-\lambda) v \quad \text { if } v=e^{\lambda t} u
$$

with (2) yields a Carleman estimate

$$
\left\|e^{\lambda t} u\right\|_{q} \leq C(p)\left\|e^{\lambda t} L u\right\|_{p}, \quad u \in C_{0}^{\infty}\left(\mathbf{R}^{n+1}\right) .
$$

Now let $W^{p}$ be the Sobolev space on $\mathbf{R}^{n+1}$ defined by the norm

$$
\left\|\left[\left(\sqrt{1+\tau^{2}}-\sigma(\xi)\right) \hat{u}(\xi, \tau)\right]^{\vee}\right\|_{p} .
$$

A short argument similar to the proof of [2, Corollary 3.1] shows that (4) implies the following: if $1<p<r$ and $u \in W^{p}$ satisfies (1) for some $V \in L^{r}\left(\mathbf{R}^{n+1}\right)$, then $u \equiv 0$ if $u$ vanishes on a half-space $\left\{(x, t): t \geq t_{0}\right\}$. This clearly yields our theorem.

The proof of Lemma 1 depends on an elementary result given below as Lemma 2. To state it we require some notation. If $\beta=\left(\beta_{1}, \ldots, \beta_{n}\right)$ is an $n$-tuple of positive numbers, define

$$
|\beta|=\sum_{l=1}^{n} \beta_{l}
$$

and, for $t>0$ and $x \in \mathbf{R}^{n}$,

$$
t^{\beta} x=\left(x_{1} t^{\beta_{1}}, \ldots, x_{n} t^{\beta_{n}}\right), \quad \frac{x}{t^{\beta}}=\left(\frac{1}{t}\right)^{\beta} x .
$$

Lemma 2. Suppose $\beta$ is as above and $f \in L^{1+1 /|\beta|}\left(\mathbf{R}^{n}\right)$. Define

$$
K(x, t)= \begin{cases}t^{-|\beta|} f\left(\frac{x}{t^{\beta}}\right) & \text { if } t>0, \\ 0 & \text { if } t \leq 0 .\end{cases}
$$

Then $K \in L^{1+1 /|\beta|, \infty}\left(\mathbf{R}^{n+1}\right)$.

Proof. The measure in $\mathbf{R}^{n}$ of the set

$$
\left\{x: t^{-|\beta|}\left|f\left(\frac{x}{t^{\beta}}\right)\right|>s\right\}
$$

is equal to

$$
\left|\left\{t^{\beta} y:|f(y)|>s t^{|\beta|}\right\}\right|=t^{|\beta|}\left|\left\{y:|f(y)|>s t^{|\beta|}\right\}\right| \doteq t^{|\beta|} D\left(s t^{|\beta|}\right) .
$$

Thus the measure in $\mathbf{R}^{n+1}$ of the set $\{(x, t):|K(x, t)|>s\}$ is

$$
\int_{0}^{\infty} t^{|\beta|} D\left(s t^{|\beta|}\right) d t=|\beta|^{-1} s^{-1-1 /|\beta|} \int_{0}^{\infty} u^{1 / \beta \mid} D(u) d u .
$$


This last integral is finite if (and only if) $f \in L^{1+1 /|\beta|}\left(\mathbf{R}^{n}\right)$.

Proof of Lemma 1. Let $r^{\prime}$ be the conjugate index of $r$. We will construct fundamental solutions $K_{\lambda}$ for the operators $L-\lambda$ on $\mathbf{R}^{n+1}$ such that

$$
\left\|K_{\lambda}\right\|_{r^{\prime}, \infty} \leq C
$$

for some $C=C(L)$ and all $\lambda<0$. Since $1 / p+1 / r^{\prime}=1 / q+1$, it will follow from Young's convolution inequality for weak $L^{p}$ (see the comment on [4, p. 121]) that

$$
\left\|K_{\lambda} * w\right\|_{q} \leq C(L, p)\|w\|_{p}
$$

for $w \in C_{0}^{\infty}\left(\mathbf{R}^{n+1}\right)$. Taking $w=(L-\lambda) u$ then yields (2). Now if $K(x, t)$ is a fundamental solution for $L$, then (3) shows that $K_{\lambda}(x, t)=e^{\lambda t} K(x, t)$ is a fundamental solution for $L-\lambda$. If $K$ vanishes for $t<0$ and if $\lambda<0$, it follows that

$$
\left\|K_{\lambda}\right\|_{r^{\prime}, \infty} \leq\|K\|_{r^{\prime}, \infty} .
$$

Thus it is sufficient to find a fundamental solution $K$ for $L$ which is in $L^{r^{\prime}, \infty}\left(\mathbf{R}^{n+1}\right)$ and vanishes for $t<0$. Let $\beta=\left(\left(2 j j_{1}\right)^{-1}, \ldots,\left(2 j j_{n}\right)^{-1}\right)$ and let $f$ be the rapidly decreasing function on $\mathbf{R}^{n}$ satisfying $\hat{f}(\xi)=e^{\sigma(\xi)}$. Define $K$ as in Lemma 2. Since $1+1 /|\beta|=r^{\prime}$, it follows from Lemma 2 that $K \in L^{r^{\prime}, \infty}\left(\mathbf{R}^{n+1}\right)$. The equation $\sigma\left(t^{\beta} \xi\right)=t \sigma(\xi)$ and a change of variables show that, for $t>0$,

$$
\widehat{K}(\cdot, t)(\xi)=e^{t \sigma(\xi)}, \quad \xi \in \mathbf{R}^{n} .
$$

It follows from this and an argument analogous to [1, Proof of Theorem 4.6, p. 195] that $K$ is a fundamental solution for $L$ on $\mathbf{R}^{n+1}$.

\section{REFERENCES}

1. G. Folland, Introduction to partial differential equations, Princeton University Press, Princeton, NJ, 1976.

2. C. Kenig, A. Ruiz, and C. Sogge, Uniform Sobolev inequalities and unique continuation for second order constant coefficient differential operators, Duke Math. J. 55 (1987), 329-347.

3. C. Kenig and C. Sogge, A note on unique continuation for Schrödinger's operator, Proc. Amer. Math. Soc. 103 (1988), 543-546.

4. E. Stein, Singular integrals and differentiability properties of functions, Princeton University Press, Princeton, NJ, 1970.

Department of Mathematics, Florida State University, Tallahassee, Florida 32306 